\title{
Therapeutic Effect of GGsTop, Selective Gamma-glutamyl Transpeptidase Inhibitor, on a Mouse Model of 5-Fluorouracil-induced Oral Mucositis
}

\author{
YOSUKE SHIMAMURA ${ }^{1}$, ISSEI TAKEUCHI ${ }^{1,2}$, HIROSHI TERADA ${ }^{1,2,3}$ and KIMIKO MAKINO ${ }^{1,2}$ \\ ${ }^{1}$ Faculty of Pharmaceutical Sciences, Tokyo University of Science, Noda, Japan; \\ ${ }^{2}$ Center for Drug Delivery Research, Tokyo University of Science, Noda, Japan; \\ ${ }^{3}$ Niigata University of Pharmacy and Applied Life Sciences, Niigata, Japan
}

\begin{abstract}
Background: Oral mucositis (OM) induced by cancer chemotherapy has a high incidence and serious symptoms, which often force chemotherapy to be stopped. GGsTop is a newly-discovered gamma-glutamyl transpeptidase (GGT) inhibitor. Previous research suggested that inhibition of GGT suppressed reactive oxygen species and induced the production of collagen and elastin. We hypothesized that GGsTop could safely treat OM. Materials and Methods: A mouse model of OM was treated with GGsTop and ulcer area, weight, and white blood cell count were determined. The treatment effect was also evaluated by hematoxylin-eosin and collagen staining. Results: The therapeutic effect of GGsTop was better than that of an existing drug and may be safely used in combination with chemotherapy. Furthermore, GGsTop promoted collagen production in oral mucosa. Conclusion: GGsTop treated OM quickly and safely. GGsTop is highly valuable for use as a treatment for $O M$.
\end{abstract}

Oral mucositis $(\mathrm{OM})$, one of the side-effects induced by chemotherapy, has reported incidence of $40 \%$, increasing to approximately $100 \%$ for chemotherapy in combination with radiotherapy $(1,2)$. OM caused by anticancer drug treatment is shown as erythema, edema or ulceration that can be accompanied by alterations ranging from mild burning sensation to large and painful ulcers and has a wider range of presentations than general stomatitis $(3,4)$. Symptoms include eating disorders, communication obstacles, and sleep disorders

Correspondence to: Kimiko Makino, Faculty of Pharmaceutical Sciences, Tokyo University of Science, 2641, Yamazaki, Noda, Chiba 278-8510, Japan. Tel/Fax: +81 471213662, e-mail: makino@rs.noda.tus.ac.jp

Key Words: Oral mucositis, chemotherapy, GGT inhibitor, wound healing, mouse model. as well as the sharp pain, which reduce the patients' quality of life (QOL) (5). Loss of consciousness was also reported to occur as a result of deterioration of $\mathrm{OM}$ which forced the treatment to stop (6). Preventing OM or treating it quickly brings improvement of QOL, and reduces the need for treatment interruption. At present, various treatments (oral cryotherapy, brushing, administration of steroidal antiinflammatory drug, disinfectant mouthwash, etc.) are performed $(5,7)$. However, they do not seem to bring enough relief nor preventive effect (8). A novel drug for OM is needed.

Anticancer drugs cause stomatitis mainly by two methods. One is by direct mucosal injury and the other is indirectly by causing a bacterial infection in the oral mucosa (9). Previous research reported that since the cell cycle of oral mucosal cells is as short as that of cancer cells, oral mucosal cells were damaged by anticancer drugs. In addition, reactive oxygen species (ROS) generated by anticancer drugs cause mucosal damage (10). It is also reported that bacterial infection is due to immunosuppression by anticancer agents, reduced mucus secretion, a decrease in the number of mucosal cells, and reduced barrier function (10). Because of these characteristics, it is impossible to use existing medicine for stomatitis, which mainly comprises steroidal anti-inflammatory drugs (11). This fact is a major cause for stomatitis during cancer chemotherapy and is regarded as a problem.

Gamma-glutamyl transpeptidase (GGT) is a highly glycosylated heterodimeric enzyme that is widely found in organisms from bacteria to mammals (12-15). GGT catalyzes the early stages of glutathione (GSH) degradation and produces cysteinyl-glycine (16). Cysteinyl-glycine is a thiol compound with very high reactivity and produces ROS which subsequently facilitates oxidative reaction $(17,18)$. GGT plays a central role in mediating the redox balance of cells, and the detoxification of xenobiotics and ROS through glutathione metabolism (19-21). Inhibition of GGT has been reported to suppress ROS, induce the production of collagen and elastin (22). 


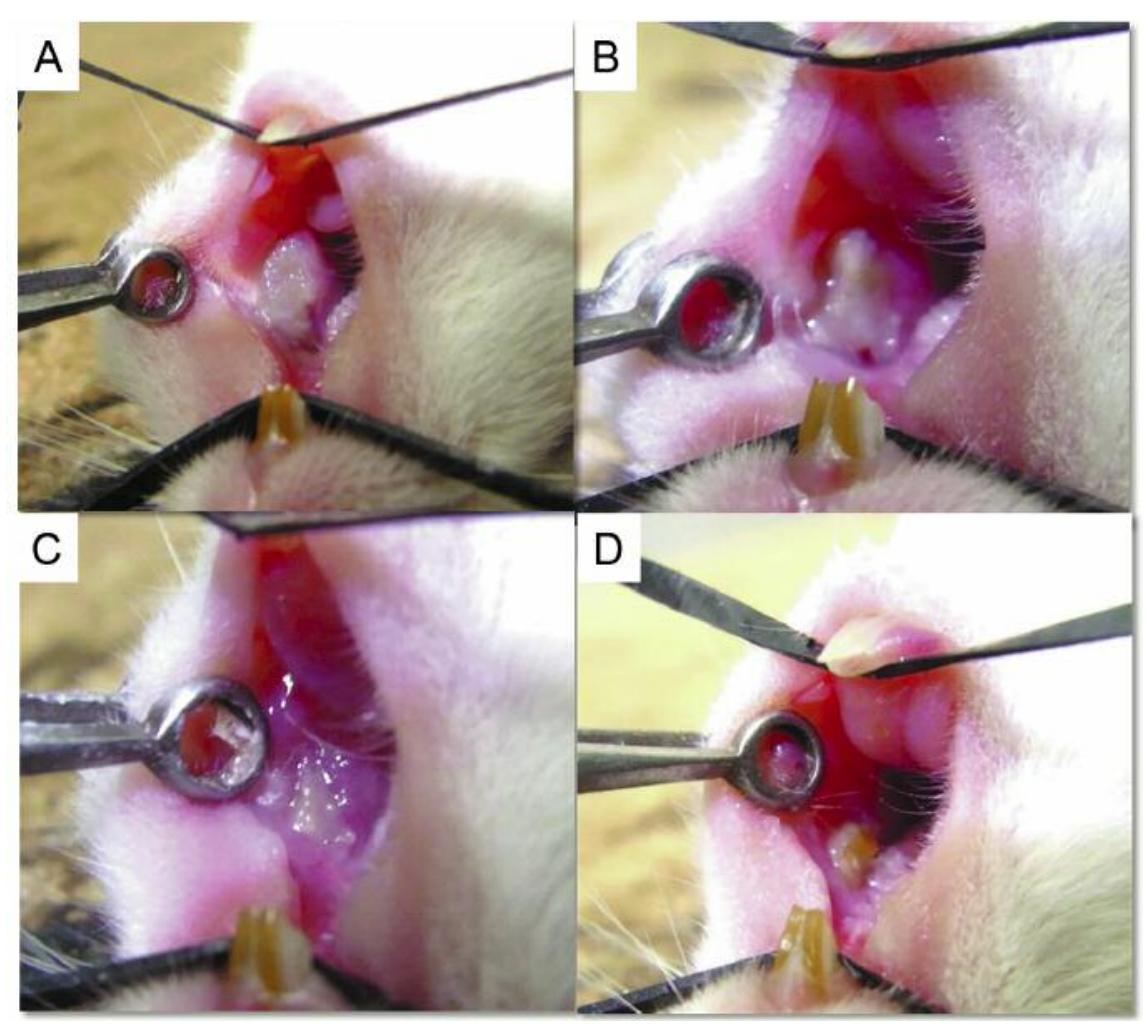

Figure 1. Oral mucositis of model mice on days $1(A), 6(B), 11(C)$, and $14(D)$.

Acivicin, L-( $\alpha \mathrm{S}, 5 \mathrm{~S})-\alpha$-amino-3-chloro-4,5-dihydro-5isoxazoleacetic acid (AT-125; produced by Streptomyces sviceus), has been used as an inhibitor of GGT (23). However, acivicin irreversibly inhibits various glutamine amidotransferases, including imidazole glycerol phosphate synthase and guanine monophosphate synthetase, and inactivates a number of biosynthetic enzymes for purine and pyrimidine, amino acids, and amino sugars (23-25), resulting in potent cytotoxicity $(26,27)$.

GGsTop $^{\text {TM }}$, 2-amino-4-\{[3-(carboxymethyl)phenoxy] (methyl)phosphoryl\} butanoic acid, is a newly discovered GGT inhibitor. GGsTop is chemically stable and nontoxic $(28,29)$. Furthermore, GGsTop exhibits activity toward human GGT of more than 100-fold that of acivicin, inhibits only GGT, and does not inhibit glutamine amidotransferases (28). In addition, GGsTop induces expression of collagen, elastin synthesis, heat-shock protein 47 , and keratinocyte induction of human skin fibroblasts $(30,31)$.

In our previous studies, 5-fluorouracil (5-FU) and acetic acid were used to prepare model mice that reproduce stomatitis developing during cancer chemotherapy (32). We hypothesized that GGsTop might safely treat stomatitis. The purpose of this study was to evaluate whether GGsTop has any therapeutic effect using model mice.

\section{Materials and Methods}

Chemicals and drug preparation. Acetic acid (purity $\geq 99.7 \%$ ) (Wako Pure Chemical Industries, Ltd., Osaka, Japan) was diluted in distilled water at 20\%. 5-FU (Wako Pure Chemical Industries, Ltd., Osaka, Japan) was diluted in saline at $8 \mathrm{mg} / \mathrm{mI}$. Turk reagent (Nacalai Tesque, Inc., Kyoto, Japan) was used for white blood cell (WBC) count. Isoflurane (Intervet Inc., Millsboro, DE, USA) was used for mouse anesthesia. Collagen stain kit (Collagen Research Center, Co., Ltd., Tokyo, Japan) was used to stain collagen in oral mucosal tissue. GGsTop was kindly provided by NAHLS. Co., Ltd (Kyoto, Japan). All other chemicals were of reagent grade.

Animals. Nine-week-old male ICR mice, weighing 30-40 g, were purchased from Japan SLC, Inc. (Shizuoka, Japan). The animals were housed in groups of five per cage in a room maintained under standardized light $(12: 12 \mathrm{~h}$ light-dark cycle $)$ at an ambient temperature of $23 \pm 2^{\circ} \mathrm{C}$, humidity of $60 \pm 10^{\circ} \mathrm{C}$ with free access to food pellets and drinking water, and were acclimated for 7 days prior to use in experiments. The experimental protocol used in this study was in accordance with the guidelines of Tokyo University of Science (approval mumber: Y18041).

OM induction. The protocol for the induction of OM was modified on the basis of a previously published protocol (32). To replicate immunosuppression induced by anticancer drug, mice were 

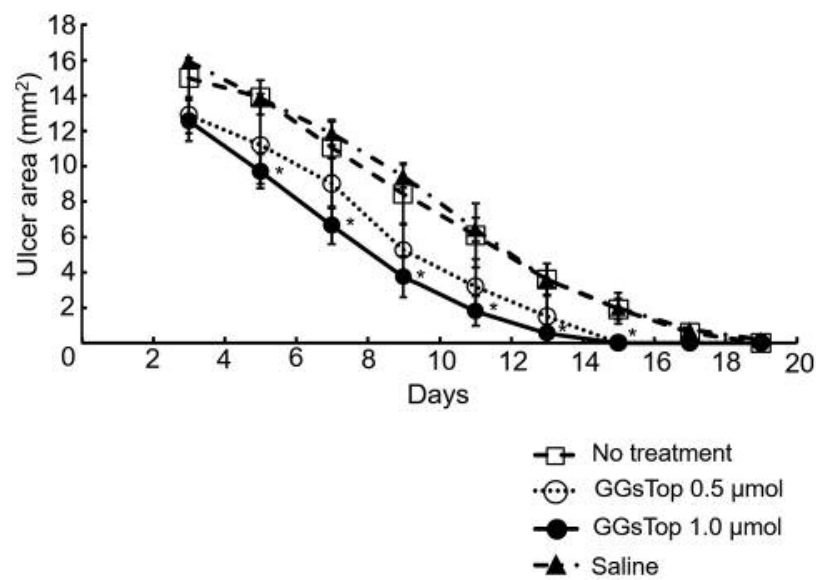

Figure 2. Changes in ulcerous area of mucosa of the left cheek of mice with oral mucositis. Data are the mean $\pm S . D$., $n=5$. *Significantly different at $p<0.05$ compared ti the group without treatment (Dunnett's test). administered an intraperitoneal injection of $5-\mathrm{FU}$ on days $-5,-3$, and -1 of the experiment at a dose of $50 \mathrm{mg} / \mathrm{kg}$ body weight. On day 0 , under isoflurane anesthesia, the left cheek of each mouse was extended outside the oral cavity. Subsequently, mucosal ulcers were induced by injection of $20 \%$ acetic acid using a microsyringe with a $31-G$ needle. Figure 1 shows the state of the oral cavity of the model mouse at intervals during the experiment.

OM treatment. From the day following the administration of acetic acid, GGsTop solution was administered to the ulcer site once with a p100 micropipette once a day. The amount of GGsTop was adjusted to be 0.5 or $1.0 \mu \mathrm{mol}$ mouse (in $33 \mu \mathrm{l}$ ). This operation was carried out until the end of complete healing, defined as the treatment period. All mice survived during the experimental period.

Assessments. From day 3 to the end of treatment, once every two or three days, the mice were anesthetized with isoflurane and the left cheek was inverted and the area of mucositis measured (as $\mathrm{mm}^{2}$ ) using image analysis software (Image J; National Institutes of Health, Bethesda, MD, USA). Furthermore, the tail venous blood was collected and diluted in Turk's reagent, and WBC count was performed with a hemacytometer.

Histological evaluation. On days 3 and 15 , oral mucosal tissue sections were produced using control mice, oral mucositis mice with OM treated with saline, and mice with OM treated with 1.0 $\mu$ mol GGsTop. Preparation of frozen tissue sections was performed based on the method of Kawamoto (33). The obtained tissue sections were examined for changes of mucosal tissues by hematoxylin-eosin staining and collagen staining. In collagen staining, red/pink color shows collagenous proteins and green color shows non-collagenous proteins.

Statistical analysis. Analysis of variance and multiple comparison tests using Dunnet's method was applied to determine differences in measures. Data are expressed as the mean $\pm \mathrm{SD}$ and differences were considered significant at $p<0.05(\mathrm{n}=5)$.

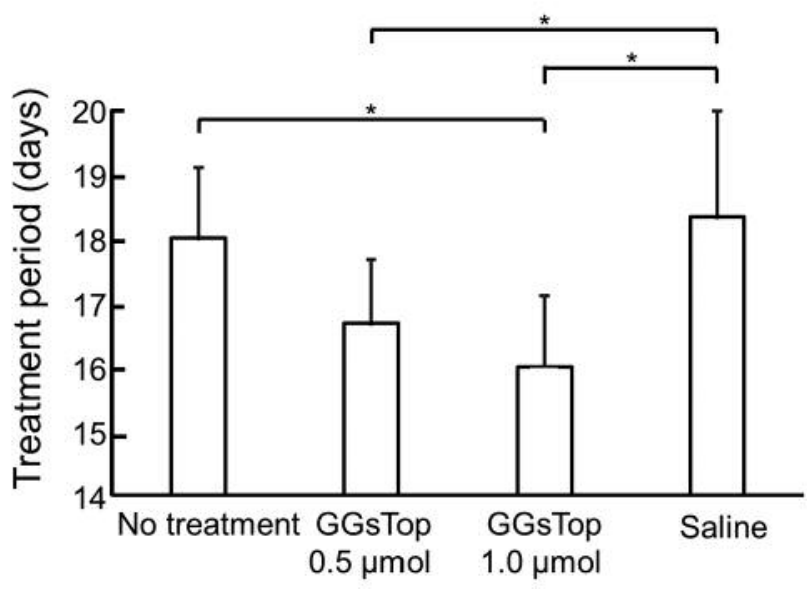

Figure 3. Treatment period of oral mucositis in modeI mice. Data are the mean \pm S.D., $n=5$. *Significantly different at $p<0.05$ compared to the group without treatment (Dunnett's test).

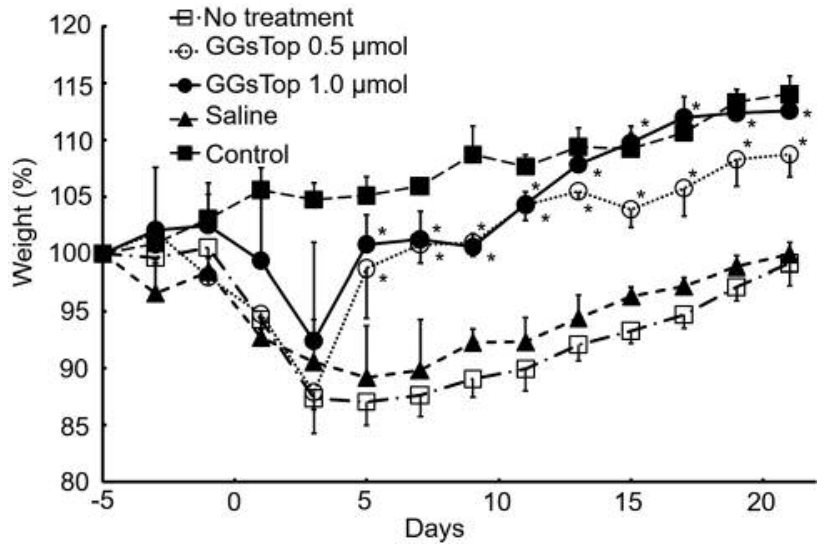

Figure 4. Change in body weight of mice with oral mucositis. Data are the mean $\pm S . D ., n=5$. *Significantly different at $p<0.05$ compared to the group without treatment (Dunnett's test).

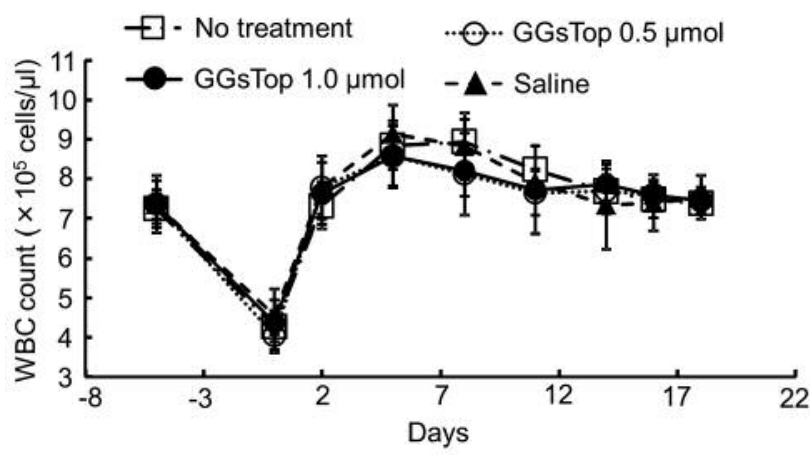

Figure 5. Changes in white blood cell (WBC) count in mice with oral mucositis. Data are the mean \pm S.D., $n=5$. *Significantly different at $p<0.05$ compared to the group without treatment (Dunnett's test). 

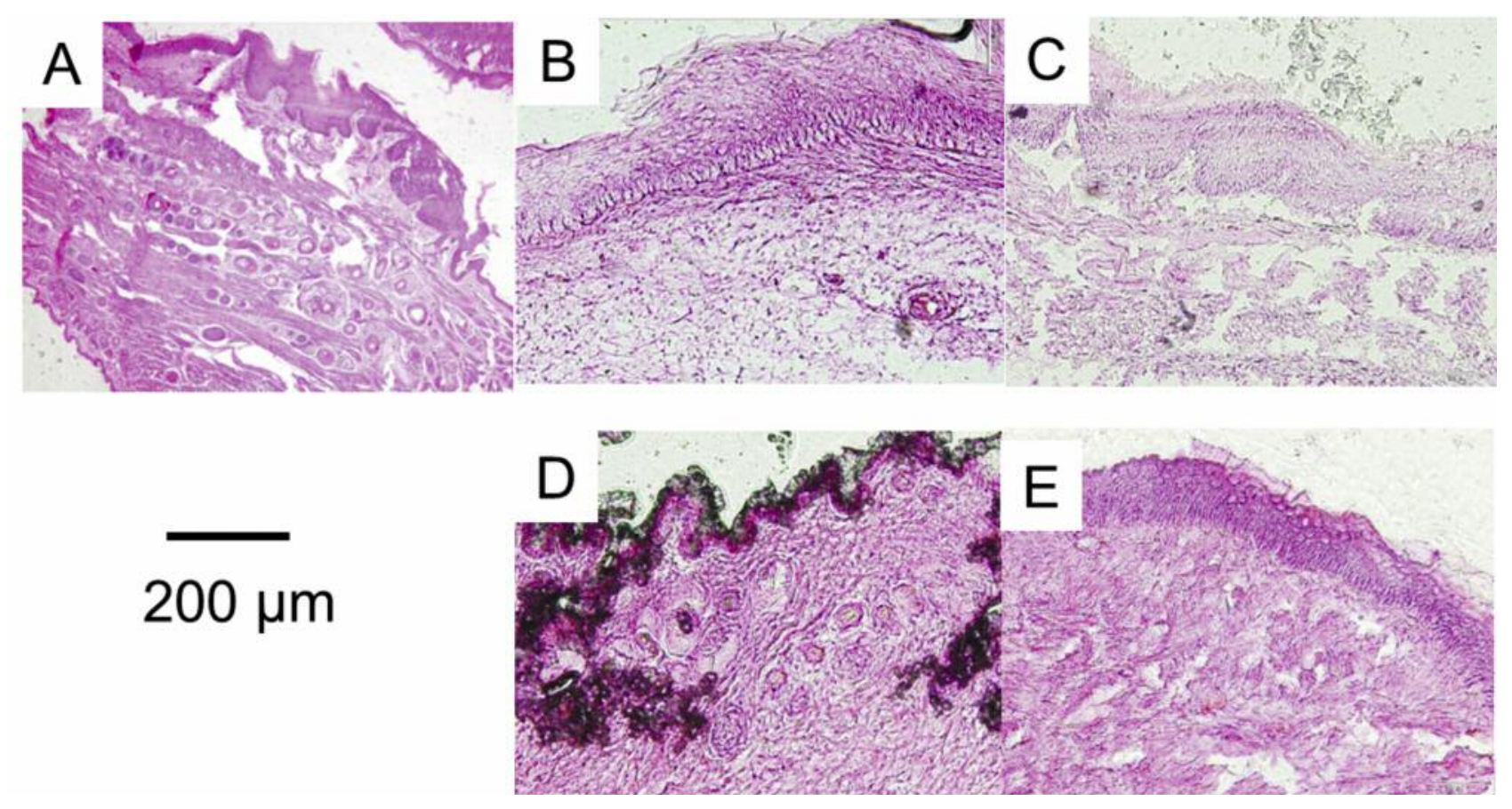

Figure 6. Hematoxylin-eosin-stained oral mucosal tissue section of normal healthy mouse (A) compared to that on day 3 and 15 in mice with oral mucositis without treatment ( $B$ and $C$, respectively) and those treated with $1 \mu \mathrm{mol}$ GGsTop (D and E, respectively).
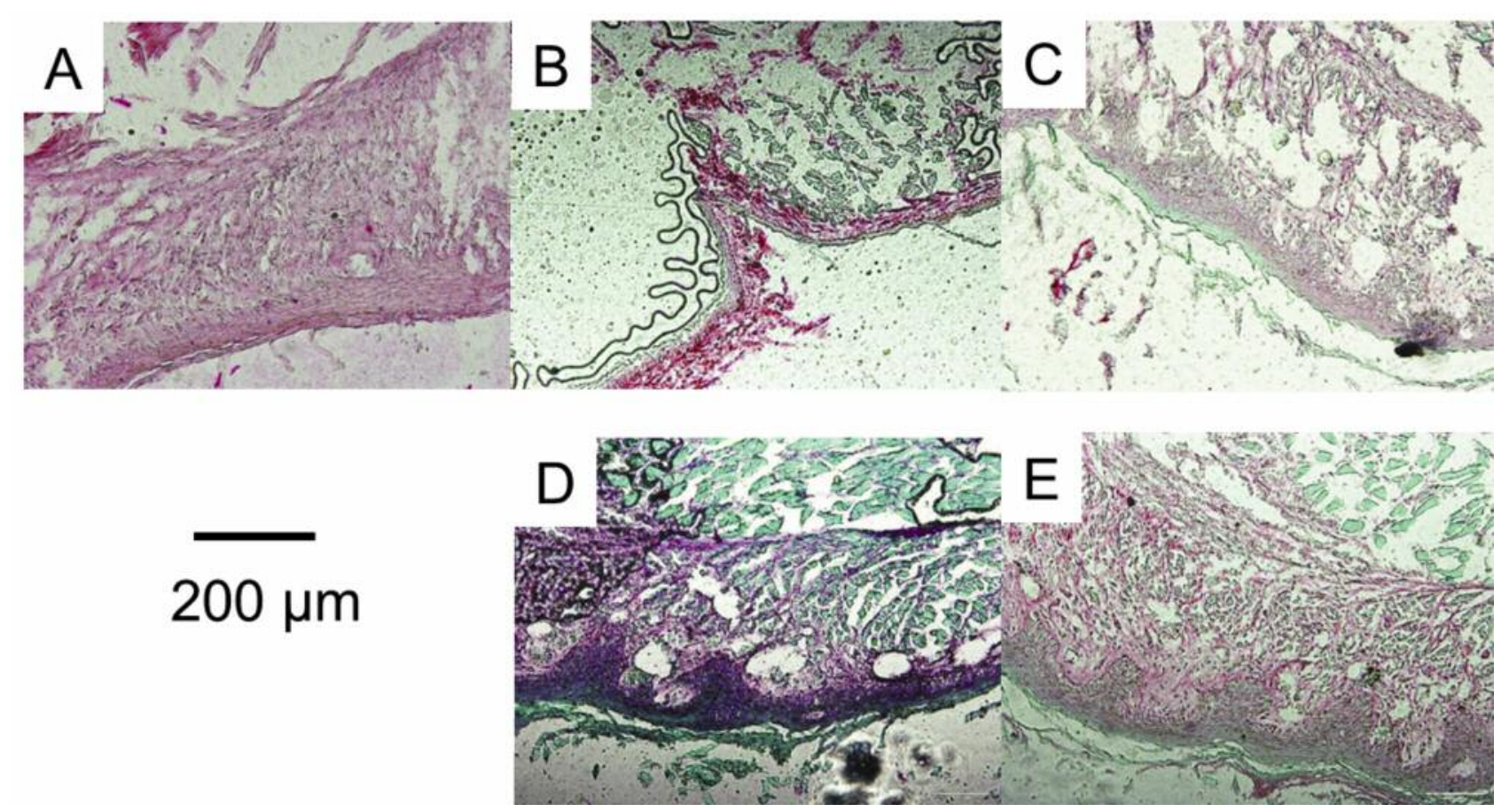

Figure 7. Collagen-stained oral mucosal tissue section of normal healthy mouse (A) compared to that on days 3 and 15 in mice with oral mucositis without treatment ( $B$ and $C$, respectively) and those treated with $1 \mu \mathrm{mol} G G s T o p$ ( $D$ and $E$, respectively). 


\section{Results}

Therapeutic effect of GGsTop on OM. Administration of GGsTop reduced the maximum ulcerated area and shortened the treatment period in a dose-dependent manner (Figures 2 and 3). Body weight was also improved by administration of GGsTop (Figure 4). The WBC count remained normal throughout the experiment in all groups (Figure 5).

Histological evaluation. HE-stained oral mucous showed GGsTop administration promoted early recovery as compared with no treatment of OM (Figure 6) Administration of GGsTop promoted collagen production in the oral mucosa (Figure 7).

\section{Discussion}

Figures 1-6 show that GGsTop not only has a therapeutic effect on stomatitis, but can also be used under immunosuppression due to pre-administration of anticancer drug. Anticancer drugs such as 5-FU are often immunosuppressive, and antiinflammatory drugs for treating stomatitis cannot be used in combination with such chemotherapy. However, since GGsTop does not have an immunosuppressive effect, it is an effective novel therapeutic drug against $\mathrm{OM}$ as a side-effect of anticancer therapy.

GGsTop is a selective GGT inhibitor. In addition, GGsTop is chemically stable and nontoxic. GGsTop has been reported to promote the production of collagen, elastin, heat-shock protein 47, and glutathione in the skin through GGT inhibition. GGsTop promotes the production of antioxidant substance, migration of epidermal keratinocytes, improvement of skin barrier function, moisturizing.

In this study, collagen production, which is most closely related to mucous membrane repair (34), was examined. Collagen is one of the main components constituting the mucosa. As the production of collagen was promoted by treatment of OM with GGsTop, mucosa damaged by inflammation was repaired quickly.

Much concerning the antioxidant action of GGsTop has been reported. GGsTop reduced oxidative stress by increasing the amount of glutathione only during inflammation and suppressed the onset of asthma (35). GGsTop prevented ischemia/reperfusion-induced renal injury by inhibiting GGT activity and ROS production $(22,36)$. From these reports, it is presumed that mucosal protection from inflammation due to antioxidant activity also applied to the stomatitis model in this study.

The result of the therapeutic effect of GGsTop was equivalent to that using Kenalog ${ }^{\mathrm{TM}}$ in the previous study (32). However, excessive reduction of WBC count occurred using Kenalog due to steroidal anti-inflammatory effect; in contrast, using GGsTop maintained a normal leukocyte number. These findings suggest that GGsTop may be a novel remedy for stomatitis.

\section{Conclusion}

In this study, GGsTop showed that it was non-toxic and had a therapeutic effect equal to or better than that of previous stomatitis remedy. Therefore, GGsTop is highly valuable for use as a treatment for OM, and further study of the treatment mechanism in detail is needed.

\section{Acknowledgements}

The Authors are grateful for GGsTop kindly provided by NAHLS. Co., Ltd (Kyoto, Japan).

\section{References}

1 Panahi Y, Ala S, Saeedi M, Okhovatian A, Bazzaz N and Naghizadeh MM: Allopurinol mouth rinse for prophylaxis of fluorouracil-induced mucositis. Eur J Cancer Care 19(3): 308$312,2010$.

2 Rubenstein EB1, Peterson DE, Schubert M, Keefe D, McGuire D, Epstein J, Elting LS, Fox PC, Cooksley C and Sonis ST: Clinical practice guidelines for the prevention and treatment of cancer therapy-induced oral and gastrointestinal mucositis. Cancer 100(9): 2026-2046, 2004.

3 Scully C, Sonis S and Diz PD: Oral mucositis. Oral Dis 12(3): 229-241, 2006

4 Chaveli-López B: Oral toxicity produced by chemotherapy: A systematic review. J Clin Exp Dent 6(1): e81-90, 2014.

5 Javadzadeh Bolouri A, Pakfetrat A, Tonkaboni A, Aledavood SA, Fathi Najafi M, Delavarian Z, Shakeri MT and Mohtashami A: Preventing and therapeutic effect of propolis in radiotherapy induced mucositis of head and neck cancers: A triple-blind, randomized, placebo-controlled trial. Iran J Cancer Prev 8(5): e4019, 2015.

6 Hurria A, Browner IS, Cohen HJ, Denlinger CS, deShazo M, Extermann M, Ganti AK, Holland JC, Holmes HM, Karlekar MB, Keating NL, McKoy J, Medeiros BC, Mrozek E, O'Connor T, Petersdorf SH, Rugo HS, Silliman RA, Tew WP, Walter LC, Weir AB 3rd and Wildes T: Senior adult oncology: Clinical practice guidelines in oncology. J Natl Compr Canc Netw 10(2): 162-209, 2012.

7 Lalla RV, Sonis ST and Peterson DE: Peterson. Management of oral mucositis in patients with cancer. Dent Clin North Am 52(1): 61-77, viii, 2008.

8 Bian L, Han G, Zhao CW, Garl PJ and Wang XJ: The role of SMAD7 in oral mucositis. Protein Cell 6(3): 160-169, 2015.

9 Lopes-Serrao MD, Ussery SM, Hall RG 2nd and Shah SR: Evaluation of chemotherapy-induced severe myelosuppression incidence in obese patients with capped dosing. J Oncol Pract 7(1): 13-17, 2011.

10 Khan SA and Wingard JR: Infection and mucosal injury in cancer treatment. J Natl Cancer Inst Monogr 2001(29): 31-36, 2001.

11 Lalla RV, Bowen J, Barasch A, Elting L, Epstein J, Keefe DM, McGuire DB, Migliorati C, Nicolatou-Galitis O, Peterson DE, 
Raber-Durlacher JE, Sonis ST and Elad S: MASCC/ISOO Clinical Practice Guidelines for the management of mucositis secondary to cancer therapy. Cancer 120(10): 1453-1461, 2014.

12 Whitfield JB: Gamma glutamyl transferase. Crit Rev Clin Lab Sci 38(4): 263-355, 2001.

13 Keillor JW, Castonguay R and Lherbet C: Gamma-glutamyl transpeptidase substrate specificity and catalytic mechanism. Methods Enzymol 401: 449-467, 2005.

14 Wickham S, West MB, Cook PF and Hanigan MH: Gammaglutamyl compounds: substrate specificity of gamma-glutamyl transpeptidase enzymes. Anal Biochem 414(2): 208-214, 2011.

15 Hanigan MH: Gamma-glutamyl transpeptidase: Redox regulation and drug resistance. Adv Cancer Res 122: 103-141, 2014.

16 Perry IJ, Wannamethee SG and Shaper AG: Prospective study of serum gamma-glutamyltransferase and risk of NIDDM: Diabetes Care 21(5): 732-737, 1998.

17 Dominici S, Paolicchi A, Corti A, Maellaro E, and Pompella A: Prooxidant reactions promoted by soluble and cell-bound gamma-glutamyltransferase activity. Methods Enzymol 401: 484-501, 2005.

18 Dominici S, Paolicchi A, Lorenzini E, Maellaro E, Comporti M, Pieri L, Minotti $G$ and Pompella A: $\gamma$-Glutamyltransferasedependent prooxidant reactions: a factor in multiple processes. Biofactors 17(1-4): 187-198, 2003.

19 Pompella A, Corti A, Paolicchi A, Giommarelli C and Zunino F: $\gamma$-Glutamyltransferase, redox regulation and cancer drug resistance. Curr Opin Pharmacol 7(4): 360-366, 2007.

20 Zhang $\mathrm{H}$ and Forman $\mathrm{HJ}$ : Redox regulation of $\gamma$-glutamyl transpeptidase. Am J Respir Cell Mol Biol 41(5): 509-515, 2009.

21 Lee DH and Jacobs DR Jr.: Is serum gamma-glutamyltransferase a marker of exposure to various environmental pollutants? Free Radic Res 43(6): 533-537, 2009.

22 Sverdrup FM, Yates MP, Vickery LE, Klover JA, Song LR, Anglin CP and Misko TP: Protein geranylgeranylation controls collagenase expression in steoarthritic cartilage. Osteoarthritis Cartilage 18(7): 948-955, 2010

23 Tamura K, Hayashi N, George J, Toshikuni N, Arisawa T, Hiratake J, Tsuchishima M and Tsutsumi M: GGsTop, a novel and specific $\gamma$-glutamyl transpeptidase inhibitor, protects hepatic ischemia-reperfusion injury in rats. Am J Physiol Gastrointest Liver Physiol 311(2): G305-312, 2016.

24 Hidalgo M, Rodriguez G, Kuhn JG, Brown T, Weiss G, MacGovren JP, Von Hoff DD and Rowinsky EK: A Phase I and pharmacological study of the glutamine antagonist acivicin with the amino acid solution aminosyn in patients with advanced solid malignancies. Clin.Cancer Res 4(11): 2763-2770, 1998.

25 Joyce-Brady $M$ and Hiratake $J$ : Inhibiting glutathione metabolism in lung lining fluid as a strategy to augment antioxidant defense. Curr Enzym Inhib 7(2): 71-78, 2011.
26 Earhart RH and Neil GL: Acivicin in 1985. Adv Enzyme Regul 24: 179-205, 1985.

27 Chittur SV, Klem TJ, Shafer CM and Davisson VJ: Mechanism for acivicin. inactivation of triad glutamine amidotransferases. Biochemistry 40(4): 876-887, 2001.

28 Han L, Hiratake J, Kamiyama A and Sakata K: Design, synthesis, and evaluation of gamma-phosphono diester analogues of glutamate as highly potent inhibitors and active site probes of gamma-glutamyl transpeptidase. Biochemistry 46(5): 1432-1447, 2007.

29 Han L, Hiratake J, Tachi N, Suzuki H, Kumagai H and Sakata $\mathrm{K}$ : Gamma-(monophenyl)phosphono glutamate analogues as mechanism-based inhibitors of gamma-glutamyl transpeptidase. Bioorg Med Chem 14(17): 6043-6054, 2006.

30 Kojima-Yuasa A, Hayashi R, Han L, Watanabe B, Hiratake J and Matsui-Yuasa I: A $\gamma$-glutamyl transpeptidase (GGT) inhibitor enhances collagen and elastin synthesis. J Jpn Cosmet Sci Soc 36(2): 93-100, 2012.

31 Watanabe B, Morikita T, Tabuchi R, Kobayashi R, Li C, Yamamoto M, Koeduka T and Hiratake J: An improved synthesis of the potent and selective $\gamma$-glutamyl transpeptidase inhibitor GGsTop together with an inhibitory activity evaluation of its potential hydrolysis products. Tetrahedron Lett 58: 3700-3703, 2017.

32 Shimamura Y, Takeuchi I, Terada $\mathrm{H}$ and Makino K: A mouse model for oral mucositis induced by cancer chemotherapy. Anticancer Res 38(1): 307-312, 2017.

33 Kawamoto T: Use of a new adhesive film for the preparation of multi-purpose fresh-frozen sections from hard tissues, wholeanimals, insects and plants. Arch Histol Cytol 66(2): 123-143, 2003.

34 Albaugh VL, Mukherjee K and Barbul A: Proline Precursors and collagen synthesis: Biochemical challenges of nutrient supplementation and wound healing. J Nutr 147(11): 2011-2017, 2017.

35 Tuzova M, Jean JC, Hughey RP, Brown LA, Cruikshank WW, Hiratake $J$ and Joyce-Brady $M$ : Inhibiting lung lining fluid glutathione metabolism with GGsTop as a novel treatment for asthma. Front Pharmacol 5: 179, 2014.

36 Yamamoto S, Watanabe B, Hiratake J, Tanaka R, Ohkita M and Matsumura Y: Preventive effect of GGsTop, a novel and selective $\gamma$-glutamyl transpeptidase inhibitor, on ischemia/ reperfusion-induced renal injury in rats. J Pharmacol Exp Ther 339(3): 945-951, 2011.

Received November 15, 2018

Revised December 2, 2018

Accepted December 4, 2018 